Cahiers de recherche sociologique

RECHERCHE

SOCIOLOGIQUE

\title{
Les amants maudits : heurs et malheurs du couple religion et politique en Europe à l'aube du troisième millénaire
}

\section{Kristoff Talin}

Numéro 33, 2000

Religions et sociétés : après le désenchantement du monde

URI : https://id.erudit.org/iderudit/1002410ar

DOI : https://doi.org/10.7202/1002410ar

Aller au sommaire du numéro

\section{Éditeur(s)}

Département de sociologie - Université du Québec à Montréal

ISSN

0831-1048 (imprimé)

1923-5771 (numérique)

Découvrir la revue

Citer cet article

Talin, K. (2000). Les amants maudits : heurs et malheurs du couple religion et politique en Europe à l'aube du troisième millénaire. Cahiers de recherche sociologique, (33), 119-147. https://doi.org/10.7202/1002410ar
Résumé de l'article

Les liens entre religion et politique sont anciens et les études les concernant, relativement nombreuses. En France, le modèle dominant des années soixante - montrant l'intensité de la relation entre vote à droite et intégration au catholicisme - est aujourd'hui remis en question. Les modèles concurrents insistent sur le pluralisme du catholicisme et sur les conséquences politiques plurielles. L'article se propose, d'une part, d'examiner la pertinence de deux modèles, et, d'autre part, de les tester en les appliquant à divers pays d'Europe. Les résultats montrent que la relation entre religion et politique demeure intense et relativement stable, quel que soit le pays étudié ou la religion prise en considération. Ainsi, même si des aménagements au premier paradigme sont nécessaires, il n'en reste pas moins que celui-ci demeure valide pour analyser le couple religion et politique. 


\section{Les amants maudits: heurs et malheurs du couple religion et politique en Europe à l'aube du troisième millénaire}

Kristoff TALIN

C'est une gageure d'écrire sur le thème religion et politique. D'une part, le sujet est si vaste qu'une vie n'y suffirait pas. D'autre part, l'étude du thème est séculaire et, depuis les pères fondateurs de la sociologie - Durkheim et Weber - qui ont bien montré l'un comme l'autre les conséquences des comportements religieux sur l'activité sociale, voire politique', les analyses se sont multipliées, si bien que le risque de redondance est élevé. Enfin, et c'est sans doute ici le risque le plus important, on peut se demander si l'étude du thème religion et politique est encore pertinente. Tant de travaux ces dernières années ont remis en cause cette articulation qu'il faut être un peu téméraire pour se risquer là où des maîtres ont déjà tout dit $^{2}$. S'inscrivant résolument dans une problématique de la modernité - travaillée par le religieux remettant en cause le désenchantement du monde, ils insistent sur l'autonomisation des croyances et la fécondation du politique par le religieux et du religieux par le politique.

S'inscrire dans cette lignée scientifique risque de rendre caduque, voire illégitime, toute recherche - un tant soit peu empirique - qui voudrait montrer que, dans la société européenne sécularisée de la fin du XXe siècle, le lien «religion et politique» a encore du sens, si ce n'est un sens.

1 É. Durkheim, Les formes élémentaires de la vie religieuse, Paris, PUF, 1922 [1912]. M. Weber, Économie et société, Paris, Plon, 1971 [1922].

J.-M. Donegani, La liberté de choisir. Pluralisme religieux et pluralisme politique dans le catholicisme français contemporain, Paris, Presses de la Fondation nationale des sciences politiques, 1993; M. Michel, Politique et religion. La grande mutation, Paris, Albin Michel, 1994; D. Hervieu-Léger, "Catholicisme et politique. Un objet sociologique à construire», Projet, no 240, 1994, p. 36-46. 
120 Religions et sociétés... après le désenchantement du monde

Pourtant, au risque de paraître archaïque, l'analyse proposée dans cet article entend montrer la permanence du modèle de Guy Michelat et Michel Simon. Certes, l'éclatement du système catholique en conséquence du concile Vatican II a permis aux catholiques de s'inscrire beaucoup plus largement sur le spectre du politique. Mais cette diffraction - si elle est indéniable et facilement observable ${ }^{3}$ reste pourtant difficilement mesurable. De plus, il ne semble pas que cette analyse invalide forcément le modèle précédemment utilisé mais qu'elle le nuance. Il s'agirait donc davantage de la modification d'un paradigme plutôt que de son changement.

Vu l'ampleur du sujet, il convient de limiter le terrain d'étude. Je ne parlerai pas de l'ensemble des religions, mais bien principalement de la religion catholique ${ }^{4}$. Des digressions seront faites, ici et là, pour examiner la pertinence de l'hypothèse dans les autres religions chrétiennes. Cet article se propose de présenter et d'analyser les modèles concurrents qui régissent l'étude du couple religion et politique, avant de montrer comment ces modèles fonctionnent dans l'espace européen ${ }^{5}$. Le lecteur l'aura compris, le sujet - religion et politique - doit être compris et restreint à la sociologie des attitudes religieuses et politiques.

\section{Religion et politique: un couple qui se déchire}

En France, lors de l'élection présidentielle de 1965, dans le cadre des premières enquêtes postélectorales, la variable religieuse est apparue comme fortement explicative des comportements politiques. La mise en lumière des liens existant entre religion et politique n'était pourtant pas nouvelle. André Siegfried avait en effet insisté sur l'importance du facteur religieux pour expliquer le «tempérament» politique de l'Ouest. «On peut dire, sans exagérer, que toutes les élections législatives depuis

\footnotetext{
3 J.-M. Donegani, ouvr. cité.
}

${ }^{4}$ Les références théoriques utilisées ici sont exclusivement, ou presque, des références françaises. D'une part, beaucoup de choses sur cette question ont été théorisées en France, d'autre part, j'aurai plaisir à soumettre son modèle de relations à l'épreuve de la comparaison.

5 Je suis bien conscient du caractère un peu ethnocentrique de mon analyse. L'absence d'éléments sur le Québec peut surprendre. Cependant, je ne suis que partiellement responsable de cette situation. L'étude des relations entre religion et politique n'est pas très développée dans les enquêtes quantitatives québécoises, si bien que les données sont pour l'instant difficilement utilisables. L'arrivée prochaine - sur le «marché» de la recherche - des données de l'International Social Survey Programme (ISSP) 1998 sur la religion devrait permettre de combler cette lacune. 
1876 se sont faites sur la question du pouvoir politique de l'Église ${ }^{6} »$. Il mettait par ailleurs l'accent sur la nécessité de distinguer le catholicisme et le cléricalisme, soulignant que «le catholicisme non clérical laisse, dans l'homme, le citoyen distinct du fidèle [...] mais [que,] dans le cléricalisme, le fidèle reconnaît au prêtre ou subit de lui une autorité politique du même ordre que son autorité religieuse, c'est-à-dire également indiscutée ${ }^{7} \gg$. Pour la première fois donc en 1965, au moment justement où la hiérarchie catholique - qui sortait du Concile Vatican II - changeait de doctrine, acceptant la liberté religieuse et reconnaissant le pluralisme politique des chrétiens, l'importance de la religion pour expliquer les attitudes politiques devenait palpable. Sur l'autel de la sociologie, la religion était intronisée «variable lourde».

\section{Religion et politique : un couple monogame}

Guy Michelat et Michel Simon ont été les premiers à montrer les liens existant entre la position de classe, l'univers religieux et l'univers politique ${ }^{8}$. L'étude a été réalisée de deux manières conjointes. D'une part, les auteurs ont mené une série d'entretiens non directifs qui ont permis d'isoler deux groupes profondément différents: les «irréligieux communisants» et les «catholiques déclarés». Dans ces deux groupes, les valeurs religieuses, tout comme les valeurs politiques, sont antagonistes. Cela aboutit à la structuration de deux systèmes de représentations. D'autre part, les auteurs ont mené une enquête quantitative auprès d'un échantillon représentatif de la population française à partir de laquelle ils ont établi une typologie distinguant quatre types d'individus. Le type A réunit des individus de gauche et faiblement pratiquants, tandis que le B est un type du centre. Au type A s'oppose le C qui est de droite et regroupe des individus massivement pratiquants'. Enfin, le type Y se caractérise par un fort apolitisme et un niveau élevé de pratique religieuse.

Par ailleurs, les relations entre intégration religieuse et attitudes politiques sont médiatisées par un système d'opinions et «un certain nombre de variables sociodémographiques, dont la classe d'appartenance et d'origine et le sexe, varient significativement d'un type à

${ }^{6}$ A. Siegfried, Tableau politique de la France de l'Ouest sous la Troisième République, Paris, Armand Colin, 1964, p. 390.

7 Ibid., p. 392.

${ }^{8}$ G. Michelat et M. Simon, Classe, religion et comportement politique, Paris, Presses de la Fondation nationale des sciences politiques et Éditions sociales, 1977.

${ }^{9}$ On constate que la pratique religieuse croît selon les types: $32 \%, 60 \%$ et $70 \%$ pour A, B et C respectivement (Ibid., p. 94). 
1'autre ${ }^{10} \gg$. Michelat et Simon constatent l'existence de corrélations entre la classe sociale objective, la classe sociale subjective, l'intégration religieuse et les attitudes politiques. Dans une démarche scientifique, les auteurs cherchent à annuler les relations entre intégration religieuse et comportement politique, mais le résultat est négatif. Ils concluent donc à l'existence de liens très puissants entre religion et politique ou, plus exactement, entre niveau de pratique religieuse et attitudes politiques. Ces liens demeurent médiatisés par l'appartenance de classe, les facteurs sociodémographiques et les systèmes d'opinions.

Il semble bien qu'en termes de probabilité (et nous insistons sur ce point) il existe une relation non seulement entre pratique religieuse et vote mais entre religion et politique au triple niveau des croyances et opinions, des sentiments et attitudes, et des comportements. Si l'on préfère, c'est bien un haut niveau d'adhésion au système symbolique du catholicisme (croyances, rituels, systèmes de normes et de valeurs, etc.) qui, dans un nombre élevé de cas, se trouve en relation avec des représentations, attitudes et comportements de droite ${ }^{11}$.

Mais, petit à petit, ce modèle est modifié ${ }^{12}$. Certes, les relations entre le vote et la pratique religieuse demeurent, mais Michelat et Simon prennent en compte l'effet du patrimoine pour formuler une explication du vote et observent des liens entre possession patrimoniale et appartenance religieuse. La possession patrimoniale augmente lorsque le degré d'intégration religieuse croît. Ils expliquent cette corrélation de deux manières. Premièrement, le fait d'être possédant accroît l'intégration au catholicisme. Cette explication structurelle, et en grande partie déterministe, est tout à fait classique dans le schéma de pensée de Michelat et Simon. L'apport essentiel de ce travail provient de l'autre explication. Il s'agit de considérer que l'appartenance au catholicisme incite davantage à la possession patrimoniale. Il y aurait une sorte «d'éthique catholique et d'esprit du capitalisme»:

Si l'on préfère, il nous paraît plus raisonnable de penser que, de façon générale, ce n'est pas le comportement d'acquisition qui entraîne une conversion religieuse (encore que le cas puisse se présenter) mais plutôt l'intériorisation de valeurs éthico-religieuses (caractéristiques de la famille, de la lignée et d'autres groupes primaires d'appartenance, et "déjà là" quand l'individu vient au monde), qui se traduit par un style de conduite

10 Ibid., p. 105.

"Ibid., p. 414.

${ }^{12}$ G. Michelat et M. Simon, «Religion, classe sociale, patrimoine et comportement électoral: l'importance de la dimension symbolique», dans D. Gaxie (dir.), Explication du vote, un bilan des études électorales en France, Paris, Presses de la Fondation nationale des sciences politiques, 1985, p. 291-322. 
Les amants maudits: heurs et malheurs du couple religion et politique 123

déterminé, favorisant une forte orientation vers la propriété patrimoniale $^{13}$.

Michelat et Simon affirment d'ailleurs que l'appartenance au catholicisme est une variable privilégiée pour expliquer les rapports entre vote, possession patrimoniale et classe sociale. Les auteurs accordent une importance beaucoup plus faible que dans leurs travaux précédents à la classe sociale, et l'intégration religieuse devient le facteur central dans l'explication des attitudes politiques. Tout se passe comme si la possession patrimoniale et l'appartenance à une classe sociale étaient un sous-ensemble de l'intégration religieuse. Elles apparaissent médiatisées, pour ne pas dire éclipsées, par l'intégration religieuse des membres. Le fondement des études sociologiques portant sur le lien entre religion et politique était posé, et il est vrai que peu d'études jusqu'au début des années quatre-vingt ont amendé ces travaux.

\section{Religion et politique: un couple tenté par l'adultère}

D'autres types de relations entre religion et politique existent. En s'intéressant à des groupes politiques ou religieux bien circonscrits, des politologues ont montré l'existence de modèles minoritaires où catholicisme et vote à droite ne vont pas forcément de pair.

Jean-Marie Donegani a publié en 1979 un article où il atteste la présence de catholiques parmi les militants du Parti socialiste ${ }^{14}$. À partir d'entretiens non directifs, il trouve, chez certains individus, un modèle d'articulation du religieux et du politique différent de celui qu'ont mis en évidence Guy Michelat et Michel Simon. Par ailleurs, chez ces militants, la pratique religieuse est faible et ne peut en aucun cas être l'indicateur synthétique d'une intégration au catholicisme ${ }^{15}$. Enfin, l'appartenance de classe semble entraîner des itinéraires différents. Les ouvriers ont été sensibilisés à l'éthique politique par la Jeunesse ouvrière chrétienne (JOC) ou par l'Action catholique ouvrière (ACO).

\footnotetext{
${ }^{13}$ Ibid., p. 305.
}

14 J.-M. Donegani, «Itinéraire politique et cheminement religieux. L'exemple de catholiques militant au Parti socialiste», Revue française de science politique, vol. 29, nos 4-5, 1979, p. 693-738.

${ }^{15}$ Voir G. Michelat et M. Simon, ouvr. cité. Dans le premier chapitre de la quatrième partie, les auteurs montrent que l'intensité de la pratique religieuse est un indicateur synthétique du degré d'intégration au catholicisme. S'appuyant sur cette observation, ils font de l'intensité de la pratique l'indicateur privilégié pour mesurer les relations entre univers religieux et univers politique. 
Ces structures permettent de concilier univers religieux et univers politique. En effet, l'engagement religieux dans les groupes de l'ACO suppose un engagement syndical. C'est un mouvement qui établit consciemment des liens entre les sphères religieuse et politique. Un chrétien, dans l'optique de l'ACO, c'est un homme qui s'engage dans le monde et qui cherche, dès aujourd'hui, à le transformer. Le rapport au politique est donc essentiellement médiatisé par le groupe. En revanche, les militants d'origine bourgeoise ont un itinéraire plus individuel. Ils ont choisi, à un moment donné, de délaisser le conservatisme religieux et politique lié à leur milieu d'origine.

Après avoir observé des socialistes chrétiens, il était assez logique de tester l'hypothèse inverse en vérifiant la présence de «catholiques de gauche» parmi les catholiques pratiquants.

Pierre Bréchon et Bernard Denni ont effectué une enquête sur les catholiques pratiquants dans la région grenobloise ${ }^{16}$. Ils montrent, à partir d'indicateurs religieux divers, que le modèle majoritaire dégagé par Guy Michelat et Michel Simon reste très présent dans le catholicisme. Toutefois, ils décèlent l'existence «d'autres modèles minoritaires d'articulation du politique, de l'éthique et du religieux ${ }^{17}$ «. Ainsi, pour les votes déclarés aux présidentielles de 1981, 35\% sont favorables à François Mitterrand et $65 \%$ à Valéry Giscard d'Estaing. Les auteurs montrent par ailleurs que l'intégration au catholicisme modifie parfois les opinions. Ainsi, au sujet du tiers-monde, les «catholiques de droite» défendent plutôt l'idée selon laquelle «la pauvreté du tiers-monde est due en grande partie à l'exploitation de ses ressources par les pays occidentaux». Or cette affirmation est contraire à ce que pense la droite en France.

\section{Religion et politique: un couple vivant la polygamie?}

La culture catholique n'est pas monolithique. Les individus sont certes plus ou moins intégrés à cette culture et, de plus, ils le sont différemment. Jean-Marie Donegani et Guy Lescanne ont réalisé une série d'entretiens non directifs pour savoir quels étaient les types de sensibilités religieuses des catholiques et quels rapports ces derniers entretenaient avec le domaine social et politique. Ils distinguent sept

${ }^{16}$ P. Bréchon et B. Denni, «L'univers politique des catholiques pratiquants. Enquête par questionnaire dans huit assemblées dominicales grenobloises», Revue française de sociologie, vol. 24, no 3, 1983, p. 505-534.

${ }_{17}$ lbid., p. 534. 
modèles d'intégration au catholicisme ${ }^{18}$. Dans quatre d'entre eux, le catholicisme n'apparait pas comme une valeur fortement structurante. Les consommateurs, les exilés, les indifférents et les culturels ne font pas référence au catholicisme comme à un des moteurs de leur existence.

Pour les consommateurs, le religieux s'exprime dans les grandes fêtes, et ils confient à l'Église le rôle d'institution présente pour les rites de passage. Être catholique, c'est alors se conformer à une évidence, c'est être comme tout le monde. La catégorie des exilés correspond à ceux qui sont malheureux dans leur identité catholique. Les auteurs parlent d'ailleurs de «conscience malheureuse». Ce sont des individus qui ont une certaine nostalgie de ce qu'ils ont connu dans leur enfance. Les indifférents, à l'opposé du type précédent, sont heureux. Que l'Église se trouve dans leur environnement ne leur pose pas spécialement de problème, elle n'exerce sur eux aucune attraction ni répulsion. Pour eux, l'Église catholique n'a pas vraiment d'importance. Enfin, les culturels sont des baptisés qui ont pris leurs distances par rapport à la norme. Ils vivent le moment présent en faisant du christianisme «une morale de l'immédiatetée ${ }^{19} »$.

Les trois autres modèles dégagés par Donegani et Lescanne peuvent être qualifiés de modèles d'intégration forte au catholicisme. Chez les individus appartenant à ces catégories, le catholicisme est en relation étroite avec la façon dont ils conçoivent et gèrent leur existence.

Les engagés sont proches de l'Action catholique et mettent l'accent sur les valeurs altruistes de la religion. Ils estiment faire partie d'un ensemble social. Engagés à la fois sur le plan social et sur le plan religieux, ils ont conscience d'appartenir à un groupe social structuré. Ils cherchent à concilier la vie et la foi qui, pour eux, ne font qu'un. De plus, ils veulent construire la vérité à partir de leur vécu. Reprenant l'éthique de l'ACO, ils pensent que les valeurs prônées par l'Évangile doivent être universelles. Leur système de représentation est fondé sur la certitude qu'il faut combattre pour changer le monde. La voie politique est sans doute l'une des possibilités pour effectuer ce changement. Elle rend possible une réalisation concrète de l'idéal évangélique selon lequel il faut plus de justice. Seuls l'incarnation dans le monde et un engagement social ou politique permettent de vivre cet idéal. Enfin, ouverts à la parole des autres, ils croient que c'est du dialogue que naîtront la justice et la vérité.

18 J.-M. Donegani et G. Lescanne, Catholicismes de France, Paris, D.D.B./BayardPresse, 1986.

Ibid., p 203. 
Pour les fidèles, tout repose sur une certitude: Dieu est. Croire en cette certitude, c'est être un homme responsable qui ne se laisse pas emporter par un groupe. La foi est un don de Dieu et la vérité n'est pas à construire, mais à recevoir. La famille occupe une place privilégiée dans leur système de valeurs. Elle incarne les valeurs morales. «Fondamentalement ce modèle n'est et ne peut être qu'individualiste. Sa tradition repose sur le primat de la personne insérée dans une communauté à structure familiale ${ }^{20} .{ }^{\prime}$ Peu enclins à la militance, cherchant plutôt à avoir un comportement exemplaire, ils s'engagent tout de même dans l'Église, car être chrétien, c'est être missionnaire. En revanche, tout engagement social est exclu. Par ailleurs, leur système de valeurs engendre la fidélité à l'Église. Il faut suivre la doctrine, car «être catholique c'est adhérer à un tout ${ }^{21} \gg$.

Le dernier modèle regroupe les fraternels. Ces personnes valorisent la réalisation de soi. Les références au présent sont nombreuses et se rattachent à la connaissance et à l'accomplissement de soi. Le respect de la personne humaine devient un absolu qu'il faut atteindre. Ainsi s'explique qu'ils se sentent plus proches des pauvres, des exclus, des marginaux. Ils n'ont pas l'âme militante, car la politique leur apparaît comme une tentation d'assistance et de démission. À leurs yeux, en effet, tout peut être accompli par soi-même. "Ma religion c'est ma $v^{22}{ }^{22} . \gg$ La référence à la communauté chrétienne est fréquente. Celle-ci semble jouer le rôle que joue la famille pour les fidèles. «Ici, la communauté n'est pas seulement, ni d'abord, la structure sociale et religieuse qui entre en cohérence avec leur vision du monde, mais c'est le lieu même qui favorise la recherche de leur identité et permet l'intégration dans un ensemble plus vaste, l'Église en particulier ${ }^{23}$.»

Ainsi, Donegani et Lescanne mettent en évidence trois formes d'intégration forte au catholicisme. Toutefois, nous l'avons vu, ces formes diffèrent. La religion apparaît donc comme une valeur fortement unificatrice, mais selon des modes différents. De plus, ils sont situés différemment par rapport à la tradition religieuse et aux valeurs politiques. L'univocité de la relation entre religion et politique est morte, vive le pluralisme! Ainsi, l'ouvrage de Jean-Marie Donegani et Guy Lescanne peut être considéré comme le nouveau paradigme définissant le couple religion et politique.

Toutefois, aussi pertinentes que soient ces analyses, je ne suis pas sûr pour autant qu'elles invalident le modèle de Guy Michelat et Michel

\footnotetext{
20 Ibid., p. 139.

${ }^{21}$ Ibid., p. 119.

22 Ibid., p. 151 .

${ }^{23}$ Ibid., p. 160
} 
Simon: l'intégration au catholicisme demeure fortement explicative du comportement politique.

\section{Religion et politique: un couple qui évolue et un mariage qui s'inscrit dans la durée}

Il semble qu'il y aurait intérêt à resituer les travaux de Guy Michelat et Michel Simon dans leur contexte. Premièrement, c'est un peu par hasard qu'ils ont découvert l'importance de la variable religieuse dans l'appréhension de l'univers politique. L'enquête de 1966, qui les amène à la mise en évidence de cette variable, visait, à l'origine, une étude des comportements politiques. Leur projet, à la différence de celui de Jean-Marie Donegani, n'était pas de faire une sociologie de l'identité catholique. On peut donc penser que les outils dont ils disposaient n'étaient pas destinés à faire apparaître la diversité du catholicisme. Il paraît bon de rappeler que l'enquête quantitative a été précédée d'une étude qualitative exploratoire dans laquelle la question qui débutait l'entretien portait sur la politique et les partis politiques et était ainsi formulée: «Pouvez-vous me dire ce que représentent pour vous la politique, les partis politiques?» C'est spontanément que certains enquêtés ont abordé la thématique religieuse. L'entretien, tout comme le questionnaire de l'enquête quantitative, ne s'articulaient donc pas à une recherche sur la diversité catholique.

Deuxièmement, nombre de sociologues ont concentré leur attention sur la dimension progressisme/conservatisme qu'ont dégagée Guy Michelat et Michel Simon ${ }^{24}$ et qui constitue le point central de leur travail: lorsque l'intégration au catholicisme croît, la probabilité d'un vote à droite augmente. Mais il me semble que leur travail ne se résume pas à cette seule proposition. Si Michelat et Simon affirment volontiers que les types $\mathrm{A}, \mathrm{B}$ et $\mathrm{C}$ forment une relation d'ordre dans un univers politique, il n'en reste pas moins qu'ils font aussi apparaître un quatrième type, appelé le type $\mathrm{Y}$, qui se caractérise par un apolitisme fort associé à un univers religieux «traditionnel». Or si beaucoup d'auteurs reprennent les modèles $\mathrm{A}$ et $\mathrm{C}$, peu utilisent le modèle intermédiaire $\mathrm{B}$ et très rares sont ceux qui gardent à l'esprit l'existence du type Y. C'est dommage, car il témoigne selon moi de la diversité politique des catholiques. L'existence d'un tel type montre que l'on peut être catholique pratiquant sans que cela s'associe forcément à un univers politique nettement conservateur. Michelat et Simon avancent alors l'hypothèse d'une moindre compétence politique du type Y. Mais

${ }^{24}$ C'est le cas de D. Hervieu-Léger, art. cité. 
on peut se demander si, en fait, il ne s'agit pas d'un modèle où la variable religieuse entraîne un retrait à l'égard de la chose politique. Autrement dit, le type $\mathrm{Y}$ ne contient-il pas en germe le modèle des «fraternels» que définiront Donegani et Lescanne quelques années plus tard? Reprendre la seule équation «intégration forte égale vote à droite» paraît un peu réducteur.

Troisièmement, Guy Michelat et Michel Simon n'affirment pas l'unicité de leurs modèles:

Ainsi les sous-cultures dont nous avons bâti les modèles à partir d'entretiens de catholiques déclarés et d'ouvriers communisants irréligieux semblent bien constituer deux pôles particulièrement significatifs pour l'analyse du comportement politique. Mais il existe, entre ces deux pôles, de multiples situations intermédiaires et, sans doute, un grand nombre de combinaisons originales dont nous sommes très loin d'appréhender la diversité et la complexité ${ }^{25}$.

Ce qu'ils mettent au jour dans leurs travaux, c'est l'existence de types majoritaires. Et rien ne dit, jusqu'à présent, que les sept modèles de Jean-Marie Donegani et Guy Lescanne s'opposent à la typologie de Guy Michelat et Michel Simon. Et si force est de constater que JeanMarie Donegani a raison quand il affirme que l'intégration religieuse par la pratique a connu un net recul $(9 \%$ à $10 \%$ de pratiquants réguliers) et que, par conséquent, le modèle de Guy Michelat et Michel Simon perd de sa valeurs - on peut en revanche se poser des questions sur la mise aux oubliettes de ce modèle explicatif ${ }^{26}$. Il semble en effet que, et ce y compris dans des travaux récents, la religion - examinée sous l'angle de la pratique religieuse - demeure une des variables lourdes de l'explication du comportement politique. Ainsi que le montrent Daniel Boy et Nonna Mayer, la variable religieuse - même si seulement un tiers des enquêtés seulement disent pratiquer au moins irrégulièrement - demeure une variable très pertinente: «Les sept variables prises en compte - degré d'activité, statut professionnel, pratique religieuse, niveau de diplômes, âge, sexe, nombre d'éléments de patrimoine - entrent toutes à des degrés divers dans les équations. Mais la religion et le statut social restent les principaux déterminants du vote pour la gauche comme pour la droite modérée. ${ }^{27}{ }^{2} \gg$

${ }^{25}$ G. Michelat et M. Simon, ouvr. cité, p. 461.

26 J.-M. Donegani, «L individu et ses credo», Projet, no 240, 1994, p. 47-55.

27 D. Boy et N. Mayer. L'électeur français a ses raisons, Paris. Presses de Sciencespo, 1997. Voir plus particulièrement leur chapitre «Que reste-t-il des variables lourdes?», p. 101-138. 
Quatrièmement, aussi clairs et indiscutables que soient les travaux montrant le pluralisme religieux et politique des catholiques, il n'en demeure pas moins qu'ils ne prennent pas en compte le poids des différents modèles dans la société française. Le peuvent-ils d'ailleurs? Est-il possible d'obtenir des indicateurs suffisamment nombreux, diversifiés et précis pour faire émerger les sept modèles de la typologie de Jean-Marie Donegani et Guy Lescanne? En fait, autant ces sept catégories apparaissent comme les types idéaux des relations qui existent entre relligion et politique parmi les catholiques, autant les groupes de Guy Michelat et Michel Simon constituent une typologie permettant de prendre toute la mesure du poids de ces relations dans la société française actuelle.

Au terme de cet examen des différents paradigmes en concurrence pour décrire, comprendre et expliquer le couple religion-politique, force est de constater que nous ne sommes pas en présence de paradigmes qui seraient en contradiction, qu'il s'agit plutôt de l'évolution d'un même paradigme. Il y a en fait, entre ces deux grands modèles, beaucoup de ressemblances et les opposer serait hasardeux. Il ne faudrait pas non plus oublier que les points de départ des auteurs ne sont pas les mêmes. Qu'y a-t-il de commun, en effet, entre la question débutant l'entretien de Guy Michelat et Michel Simon et celle de JeanMarie Donegani? Alors que la première portait sur la politique et les partis, la seconde invitait à une réflexion sur le fait d'être catholique.

La suite de cet article voudrait ainsi apporter une modeste contribution au vaste débat inauguré par nos prédécesseurs. Il s'agit de montrer comment, dans un certain nombre de pays européens, religion et politique - au singulier - forment encore un modèle d'analyse pertinent pour la compréhension des comportements individuels. Nous verrons par la même occasion que l'équation «intégration religieuse forte égale univers politique conservateur» demeure valide et qu'elle s'applique aussi à la religion protestante, voire orthodoxe. À cette intention, je me servirai des données des enquêtes Eurobaromètre ${ }^{28}$.

\section{Un mariage qui déborde les frontières}

Parler du lien entre religion et politique en tant que tendance, fréquence et positionnement des individus est certes au cœur du sujet tel que j'ai voulu l'aborder. Le lecteur avide de détails vivra alors des frustrations.

${ }^{28}$ Les enquêtes Eurobaromètre sont financées par l'Union européenne à des fins de connaissance politique de l'opinion publique européenne. Ce ne sont donc pas des enquêtes de recherche, mais leurs résultats sont utiles aux chercheurs. 
L'intrusion dans la chambre à coucher est-elle légitime?

Ledit lecteur pourra regretter l'absence de mise en perspective en ce qui concerne le continent nord-américain, ou bien encore il pourra me reprocher l'imprécision des concepts ou leur manque de pertinence. Il pourra ensuite critiquer ma position épistémologique qui privilégie la régularité de la relation à la singularité de l'événement. Face à ces critiques légitimes, le sociologue que je suis implore la clémence du lecteur et tentera d'expliciter son propos.

1. D'une part, le but de ce texte est de montrer le changement de paradigme théorique dans l'étude du couple religion et politique. D'autre part, il s'agit de tester les paradigmes à l'échelle de l'Europe. L'ampleur du sujet est déjà telle que des choix draconiens et cornéliens ont été indispensables. Qui plus que le sociologue de la religion empiriste n'aurait pas désiré avoir un échantillon - ou mieux, un panel - permettant la comparaison dans le temps et dans l'espace sans se soucier de la taille de son échantillon. Mais les limites de l'exercice tiennent précisément à sa nature. Entrer dans un champ de validation popperien et essayer de faire œuvre scientifique, c'est faire face aux réalités inhérentes à l'empirie: les données ont leurs limites et il est souvent obligatoire d'utiliser des données imparfaites.

2. On pourrait objecter que l'étude menée ici est fallacieuse parce que l'indicateur qu'est la pratique religieuse n'a pas la même signification pour toutes les religions. Il serait donc illusoire de vouloir mener une étude comparative sur plusieurs religions. Deux arguments permettent au moins de relativiser cette objection. Le premier est que les religions que je compare sont toutes chrétiennes. Comme elles ont des racines et une histoire européenne communes, on peut penser qu'elles se prêtent alors à la comparaison. Le second argument est que si le choix de l'indicateur, c'est-à-dire la pratique, est discutable, celle-ci n'en demeure pas moins un indicateur solide. Dans un espace géographique ayant une histoire commune et en considérant des religions ayant un terreau commun, on peut tout de même croire que cet indicateur est pertinent, quelle que soit la religion chrétienne européenne prise en considération. De plus, je n'ai pas l'intention de me livrer à une déconstruction théorique quant à la pertinence de cet indicateur dans une perspective comparative, l'intérêt de l'analyse portant sur un autre élément: il s'agit de déterminer si les conséquences d'un degré donné d'intégration à une religion sont les mêmes sur le plan politique.

3. Il est relativement fréquent de s'intéresser à la désinstitutionnalisation du croire ou de montrer comment le pluralisme produit des formes politiques singulières, et je ne renie pas l'extrême richesse de 
ces études. Cependant, telle n'est pas ma perspective. Je veux plutôt prendre la mesure de la continuité des liens entre religion et politique et montrer la permanence de la relation dans l'espace européen. Cette prise de position épistémologique met en avant la continuité plus que la discontinuité et je veux analyser ainsi plus sous l'angle des transformations que des ruptures les changements contemporains. Cette perspective met de plus l'accent sur les mutations externes des relations entre religion et société, alors qu'une épistémologie plus singulière s'attachera davantage aux mutations internes touchant la sphère religieuse vues comme une conséquence de la modernité.

Tester le modèle de Guy Michelat et Michel Simon dans l'espace européen, en vérifier la pertinence dans différents pays, différentes religions et mesurer sa variation dans le temps, tel est l'objectif. Pour ce faire, ma base de travail sera essentiellement les données cumulées des enquêtes Eurobaromètre.

Pour ne pas compliquer l'analyse et de façon à tenir compte des contraintes liées à la production même des données, il m'a fallu faire certains choix méthodologiques. Par exemple, afin de pouvoir effectuer une analyse satisfaisante de la pratique religieuse, j'ai dû la recoder en distinguant simplement les pratiquants des non-pratiquants. De la même manière, pour ne pas multiplier les indicateurs politiques, j'ai choisi de me référer à un indicateur synthétique: l'échelle gauche-droite.

Un couple stable pendant près de vingt ans (1973-1992)

D'abord, on observe une grande stabilité dans le temps de la structure du couple religion-politique (voir le tableau 1). Indépendamment de la religion ou de l'année considérées, les non-pratiquants sont toujours positionnés à gauche sur l'échelle gauche-droite, sauf en 1988 où les protestants pratiquants sont plus nombreux à gauche que les non-pratiquants.

On note aussi que l'écart entre pratiquants et non-pratiquants se réduit chez les catholiques. Observer le même type de relation chez les protestants permet d'insister sur les conséquences de l'intégration à un système normatif. Parallèlement, on peut en déduire que la corrélation entre le fait de ne pas pratiquer un positionnement à gauche est moins forte dans les années quatre-vingt-dix que dans les années soixante-dix.

Plusieurs hypothèses explicatives peuvent être avancées. Premièrement, ce resserrement découle de l'évolution des pratiquants. Dans le cas des catholiques - moins proches de la norme de l'Église en 1992 que vingt ans auparavant, encore pratiquants, mais de moins en 
moins régulièrement -, il est légitime de penser qu'ils ont pris leurs distances par rapport au conservatisme politique, et ce même chez les catholiques les plus pratiquants. Il est probable que le concile Vatican II, en légitimant le pluralisme, a contribué à l'émancipation politique du catholique. Deuxièmement, la société européenne ayant beaucoup changé entre 1973 et 1992, les motivations qui poussent au positionnement à gauche ont évolué. Dans le contexte contestataire des années soixante-dix, l'ordre établi était remis en question et la dimension politique devenait le moteur de l'engagement religieux. Par exemple, on était des «cathos de gauche» non pratiquants. Dans la décennie 1990, les valeurs changent et l'appartenance à un groupe religieux devient prépondérante, le positionnement politique passant au second plan. Par exemple, il est plus important de s'affirmer protestant - donc adhérant à une religion - que de se dire «protestant de gauche» ou «protestant de droite». Bref, l'affirmation d'une religion prédomine sur l'affirmation d'une position politique.

Enfin, on peut se demander si la fin des idéologies et la quasidisparition du système communiste n'ont pas amoindri l'opposition idéologique qui existait entre pratiquants et non-pratiquants ou entre pratiquants et sans-religion.

Par ailleurs, le «rapport de chance» (odds-ratio) en ce qui concerne le vote pour un parti de droite ou de gauche en lien avec la pratique religieuse décroît régulièrement (voir le tableau 2 ).

Cela signifie, si l'on envisage une analyse longitudinale, que les liens entre l'intégration religieuse forte et le vote à droite sont de moins en moins intenses. Il n'en demeure pas moins que l'intégration religieuse demeure toujours une variable explicative lourde $\mathrm{du}$ comportement politique ${ }^{29}$. Selon le paradigme, on pourra montrer la pertinence ou la non-pertinence du couple religion et politique. Quoi qu'il en soit, cette analyse longitudinale indique clairement que la relation entre religion et politique opère de la même manière dans les trois religions chrétiennes. Du coup, on peut tenter d'étendre le modèle élaboré par Guy Michelat et Michel Simon. À la lumière de mes observations, il semble en effet possible de dire que la relation demeure vérifiable pour d'autres religions que la religion catholique. Ce n'est donc pas seulement une intégration au catholicisme qui déterminerait des comportements politiques conservateurs, mais bien l'intégration à une religion instituée. À partir du moment où une religion quitte la voie prophétique et contestataire pour devenir «attestaire», on peut se demander si le conservatisme politique ne va pas de soi. Dès lors, c'est

${ }^{29}$ Une étude effectuée avec l'échelle gauche-droite donne des résultats similaires. Les relations entre pratiquants et sans-religion se nouent de la même manière. 
moins le contenu dogmatique de la religion considérée qu'il faut prendre en compte que son attestation politique. Toute religion qui perdrait son caractère prophétique se trouverait donc potentiellement porteuse de schèmes politiques conservateurs. Cette hypothèse mérite d'être vérifiée, mais, dans un premier temps, il convient de la tester dans différents pays européens. En effet, il se pourrait fort bien que la relation existe dans un contexte national précis, mais qu'elle ne soit pas significative dans un pays biconfessionnel, par exemple.

Avant de tenter d'expliquer la diversité des situations selon les pays, je veux montrer quelques aspects généraux de la relation religionpolitique (voir le tableau 3). Premièrement, dans chacun des 11 pays pris en considération ${ }^{30}$, les non-pratiquants se situent toujours plus à gauche que les pratiquants. Il existe donc une certaine universalité du couple religion-politique.

Par ailleurs, si l'écart entre pratiquants et non-pratiquants est globalement plus grand chez les catholiques $(17,2 \%)$ que chez les protestants $(13 \%)$, il n'en demeure pas moins que, pour l'ensemble, les différences sont d'importance. Même les orthodoxes confirment le sens et l'intensité de la relation $(27 \%)$. Pour le catholicisme, l'écart entre pratiquants et non-pratiquants fluctue, mais il reste élevé, quel que soit le pays. L'Italie, avec $30 \%$, présente l'écart maximal, ce qui s'explique très bien. Dans ce pays encore très marqué par le catholicisme et où l'on enregistre un haut niveau de pratique religieuse hebdomadaire, ne pas aller à la messe, c'est faire des choix de contestation - ecclésiale et politique - assez nets. Il n'est donc pas très surprenant que les catholiques non pratiquants soient beaucoup plus à gauche que les pratiquants. Le cas du Portugal, le pays où l'on relève le moins d'écart de positionnement politique entre les catholiques pratiquants et les catholiques non pratiquants, est plus complexe à analyser. Deux hypothèses peuvent être avancées pour expliquer ce phénomène. D'une part, l'influence de l'Église varie beaucoup selon les régions et il est possible que les variations régionales se traduisent par une moindre différenciation des attitudes politiques. D'autre part, la situation concordataire en vigueur au Portugal peut contribuer à amenuiser les différences entre catholiques pratiquants et non pratiquants.

L'autre observation notable dans ce tableau, c'est le cas des sansreligion. Quel que soit le pays étudié, les sans-religion sont toujours plus à gauche que les non-pratiquants. Que le pays soit de tradition orthodoxe, protestante ou catholique, cette tendance varie peu. Ainsi, en Grèce, pays orthodoxe, l'écart entre les sans-religion et les nonpratiquants est de $34 \%$, alors qu'il est de $12 \%$ dans un pays protestant

${ }^{30}$ Le Luxembourg n'a pas été pris en compte à cause de sa faible population. 
134 Religions et sociétés... après le désenchantement du monde

comme le Danemark et de $16 \%$ dans un pays catholique comme l'Italie.

Les hypothèses de départ - testées dans une perspective rétrospective - trouvent donc une relative confirmation. L'influence de la variable religieuse sur l'orientation politique demeure forte. Si ce résultat n'est pas une surprise dans le cas des catholiques ou des orthodoxes, il jette une nouvelle lumière sur le positionnement politique des protestants. L'idée d'un protestantisme éclairé et ayant mieux assimilé les valeurs de la modernité politique que le catholicisme plus à gauche ne fait pas recette.

Les sans-religion sont, dans tous les cas de figure, les moins conservateurs. Il est bon aussi de rappeler que l'écart - au chapitre de l'orientation politique - entre les pratiquants, les non-pratiquants et les sans-religion tend à s'amenuiser au fil des années, ce qui témoigne de la recomposition des systèmes de valeurs des Européens.

Un couple livré aux mutations: le point en 1998

La relation entre religion et politique ayant été mise en perspective, il convient à présent d'analyser la situation actuelle. À partir des données de l'Eurobaromètre 50 de 1998, c'est une actualisation de la réflexion que je propose. Comment, sous l'impact de la sécularisation et de la déchristianisation, et avec la perte d'influence des Églises, le couple religion et politique évolue-t-il? Que se passe-t-il au sein de l'Union européenne à la suite de l'arrivée d'un nombre grandissant de pays?

En 1998, dans l'Union européenne, les catholiques formaient plus de $50,7 \%$ de la population, loin devant les protestants $(16,7 \%)$ et les orthodoxes $(3,5 \%)$. Les autres religions ne représentaient que $4,4 \%$ de la population. La principale modification, ces dernières années, vient de la montée en puissance du groupe des sans-religion, qui a progressé régulièrement pour atteindre, en $1998,24,6 \%$ de la population européenne. On a donc à la fois l'impression d'une grande stabilité, mais aussi d'une forme religieuse de New Deal avec la montée de l'indifférence religieuse.

Le premier élément qui ressort dans le tableau 4, c'est la grande convergence de ces données et de celles des années précédentes ( $c f$. tableau 1). En ce qui concerne la moyenne européenne de ceux qui se placent à gauche de l'échiquier politique, on note une légère progression (3\%), mais ce qui prédomine, c'est d'abord la permanence. Comme précédemment, lorsqu'on est pratiquant, on est toujours moins 
à gauche que lorsqu'on est non pratiquant. Par ailleurs, l'écart entre protestants et catholiques s'amenuise, si bien que la position politique des uns et des autres est assez proche. Ce que l'on affirme pour le protestantisme et le catholicisme n'est en revanche pas fondé pour l'orthodoxie. L'écart entre pratiquants et non-pratiquants demeure plus net que dans les autres religions. L'autre élément que met en évidence ce tableau, c'est le resserrement des écarts entre les non-pratiquants et les sans-religion. La tendance observable pour le catholicisme depuis plusieurs années devient décelable pour les autres religions. La proximité entre les sans-religion et les non-pratiquants s'accompagne d'une légère distanciation entre les pratiquants et les non-pratiquants.

Les résultats présentés dans le tableau 5 permettent de mesurer les écarts entre la tendance au cours des années 1975 à 1992 et la situation actuelle. Si l'on est attentif aux résultats globaux de ce tableau, le premier constat qui s'impose, c'est la permanence de la relation. Ici aussi, quel que soit le pays pris en considération, les pratiquants sont toujours moins à gauche que les non-pratiquants, eux-mêmes moins à gauche que les sans-religion. Toutefois, certaines nuances viennent relativiser cette vision d'ensemble. Ainsi, en Belgique, les nonpratiquants sont moins nombreux à gauche que les pratiquants. Mais ce phénomène s'explique peut-être par le contexte particulier de la Belgique au cours des quatre ou cinq dernières années. Les scandales sexuels, et surtout politiques, ont provoqué un sérieux traumatisme par rapport à la politique et il est possible que l'inversion de la relation soit liée à des facteurs plus conjoncturels que structurels.

Derrière la permanence de la relation, on voit se modifier sensiblement son intensité. Quelle que soit la religion considérée, chaque pays - à l'exception de l'Allemagne et du Portugal - voit les positions politiques des pratiquants et des non-pratiquants se rapprocher et l'écart varie de $1 \%$ pour l'Espagne $17 \%$ pour la Belgique. Le cas de l'Allemagne peut probablement s'expliquer par le processus de réunification qui a sans doute bousculé les cartes politiques et religieuses du pays. Quant au Portugal, on voit l'écart s'accroître entre pratiquants, non-pratiquants et sans-religion sans que des explications très claires apparaissent. Tout au plus peut-on émettre l'hypothèse que la montée régulière des forces de gauche depuis dix ans engendre des positions politiques plus tranchées entre catholiques pratiquants, catholiques non pratiquants et sans-religion.

Par ailleurs, dans deux des pays biconfessionnels - la GrandeBretagne et l'Allemagne -, l'écart entre les sans-religion et les catholiques non pratiquants est négatif. Autrement dit, les sans-religion sont moins à gauche que les catholiques non pratiquants. Dans deux pays où le catholicisme ne compose pas le terreau culturel du pays, on 
peut supposer que le fait de se dire catholique est déjà un positionnement social plus affirmé que dans un pays où être catholique est la norme. Dans ces conditions sociétales, être catholique non pratiquant, c'est faire preuve d'une double autonomie. Autonomie d'une part par rapport à la société en se disant catholique, et d'autre part par rapport à l'Église catholique en s'affirmant non pratiquant. Il n'est donc pas très surprenant que les catholiques non pratiquants anglais ou allemands soient plus à gauche que les sans-religion.

Pourquoi la mariée n'est-elle pas en noir?

Dans l'étude du couple religion-politique, la question qui brûle les lèvres est souvent celle qui porte sur les situations extrêmes. Si un fort degré d'intégration religieuse augmente la probabilité d'avoir un univers politique conservateur, on peut penser qu'il existe une forte corrélation entre une intégration religieuse maximale et un univers extrémiste de droite. Or il n'en est rien. Des politologues ont observé que l'intégration à la religion catholique protège d'un attrait pour l'extrême droite ${ }^{31}$ et les raisons qui l'expliquent sont assez claires. Une de ces raisons réside dans le fait que la position des évêques catholiques par rapport aux idées de l'extrême droite est sans ambiguité. Ainsi, dès la naissance du Front national (FN), l'épiscopat a rappelé les exigences évangéliques et souligné leur incompatibilité avec les thèses du FN et l'on peut penser alors que la prise de position des évêques a porté ses fruits. Par ailleurs, si certains catholiques ont eu des tentations extrémistes, ils doivent être très minoritaires. Il ne s'agit pas de nier l'existence de groupes traditionalistes intégristes ayant choisi de voter pour Le Pen et d'adopter le rite de saint Pie V, mais simplement de souligner leur faible nombre. Enfin, on notera avec un certain soulagement qu'une étude temporelle montre qu'au fil des années la part des catholiques dans l'électorat d'extrême droite a tendance à diminuer. «De 1984 à 1997, le FN a perdu 7 points chez les catholiques pratiquants réguliers et en a gagné 12 chez les sans-religion ${ }^{32} . »$ On manque de recul à l'échelle européenne pour pouvoir être affirmatif, mais il est permis d'imaginer le même type d'antinomie entre extrême droite et degré élevé d'intégration religieuse. En tout cas, on peut affirmer que les enquêtes quantitatives ne montrent pas l'inverse. Dans une société européenne où se manifeste une montée des nationalismes, il était important de le souligner.

${ }^{31}$ P. Perrineau, Le symptôme Le Pen. Radiographie des électeurs du Front National, Paris, Fayard, 1997: N. Mayer. Ces Français qui votent FN, Paris, Flammarion, 1999.

${ }^{32}$ P. Perrineau, ouvr. cité. p 112. 
Mais il semble indispensable de montrer, pour compléter ce panorama, comment le religieux et le politique peuvent se lier d'une manière particulière au sein de groupes politiquement ou religieusement bien identifiés. Les groupes qui sont présentés dans la suite doivent être considérés comme des idéaux-types de modes d'articulation bien spécifiques entre religion et politique.

\section{Des couples aux mœurs particulières}

Agnès Rochefort-Turquin s'intéresse à un mouvement de socialistes chrétien $^{33}$. Ces chrétiens, qui semblent appartenir au courant «intégral» et «intransigeant» du catholicisme, ont décidé, à la fin des années vingt, de s'engager en politique. Ils sont principalement issus de couches sociales populaires. Leur leader, Maurice Laudrain, cherche à rapprocher les idées chrétiennes et socialistes. En 1935, un mouvement voit le jour, le Front uni des chrétiens révolutionnaires. Les membres animent un journal, Terre nouvelle, qui affirme ouvertement le désir de conciliation entre socialistes et chrétiens. Il scandalisera l'opinion, car il fait référence explicitement au communisme ${ }^{34}$. Les réactions de la hiérarchie catholique face à Terre nouvelle sont très vives. En juillet 1936, le journal sera mis à l'Index par le Vatican. Le mouvement valorise l'action politique, qu'il conçoit comme un service à rendre à la collectivité. La ligne du mouvement est simple: tout chrétien sincère se doit d'être un révolutionnaire et de se dresser contre le capitalisme. À ses yeux, le christianisme est vécu de façon inconsciente par les travailleurs en lutte. L'adhésion de ces chrétiens au Front populaire sera massive. Ils comptent sur la présence des communistes dans le mouvement, tout en rappelant l'incompatibilité entre être chrétien et adhérer au Parti communiste. Ce mouvement n'est pas monolithique. Dès la création du Front populaire, des divergences apparaissent entre «pacifistes» et «révolutionnaires». Ces deux tendances donneront par la suite les «munichois» et les «anti-munichois». Les «munichois» refusent l'union nationale, alors que les «anti-munichois» sont prêts à l'accepter pour lutter contre le fascisme. L'auteure montre donc que l'affirmation chrétienne engendre parfois des attitudes politiques contestataires. Comme pour les études sur les itinéraires individuels, les liens entre les croyances religieuses et les attitudes politiques ne sont donc pas systématiques. Une forte intégration religieuse n'engendre pas nécessairement un comportement politique conservateur.

${ }^{33}$ A. Rochefort-Turquin, Front Populaire: socialistes parce que chrétiens, Paris, Cerf, 1986.

${ }^{34}$ Une faucille et un marteau sur fond de croix chrétienne illustrent la couverture du journal. 
Dans un livre portant sur l'histoire des chrétiens dans le monde rural $^{35}$, les auteurs montrent comment les objectifs du mouvement ont évolué au fil des années. Le mouvement naît en 1939 sous le nom de Ligue agricole catholique (LAC). L'impulsion de départ est une rechristianisation de la France rurale. Pendant la guerre, le mouvement s'oriente essentiellement vers une pastorale familiale avec la diffusion du journal du mouvement, Foyer rural. À la fin de la guerre, la LAC devient le Mouvement familial rural (MFR) et garde, comme son nom l'indique, la famille comme axe prioritaire. Ce changement de nom témoigne d'une volonté du mouvement de se démarquer des mouvements de l'Action catholique. Dans l'après-guerre, un changement s'amorce. On veut toucher un large public rural, mais aussi inciter les membres du mouvement à adhérer aux organisations qui se développent dans le monde rural. On note donc, au cours de cette période, une volonté de quitter le giron ecclésial pour s'insérer dans le monde. Le mouvement, conscient de la diversification du monde rural, se structure en créant des réseaux socioprofessionnels. À partir du concile, le MFR, qui deviendra Chrétiens en monde rural en 1966, prend un tournant. En 1965, le Congrès de Chartres ébranle les certitudes fondatrices du mouvement. D'une part, à la suite du concile, l'Église n'a plus l'uniformité d'autrefois. Elle n'est plus détentrice d'une vérité inaliénable et dogmatique. D'autre part, le monde n'apparaît plus comme étranger. Dieu y est présent avec les hommes. "Construire le monde, c'est participer à la création par Dieu ${ }^{36} . \gg$ De plus, le mouvement redéfinit sa mission. La rechristianisation n'est plus évoquée, mais le mouvement insiste sur l'apport du monde pour les chrétiens et l'apport des chrétiens pour la transformation du monde. Enfin, en 1972, le mouvement rompt avec le passé. Deux orientations sont prises. La structuration en réseaux socioprofessionnels s'intensifie et chaque réseau bénéficie désormais d'une large autonomie. L'autre orientation concerne l'esprit du mouvement qui affirme une volonté de combattre, au nom de l'Évangile, pour les grands problèmes humains. Il s'agit là d'une volonté affirmée d'articuler le mouvement et la société. C'est donc l'évolution de l'éthique prêchée par le mouvement qui nous intéresse ici. Ce mouvement d'action catholique, bien qu'il soit spécialisé dans un milieu assez conservateur, est marqué par l'évolution du monde et de l'Église. On voit ainsi qu'une forte intégration religieuse, dans un milieu plutôt conservateur, n'engendre pas nécessairement des attitudes religieuses et sociales conservatrices.

André Grelon et Françoise Subileau établissent une comparaison entre les membres du Mouvement des cadres chrétiens (MCC) et ceux

${ }^{35}$ J.-L. Ducasse et autres, Chrétiens dans le monde rural, Paris, Éditions ouvrières, 1989

Ibid., p. 147. 
de La Vie nouvelle ${ }^{37}$. Les premiers se rapprochent du modèle de forte intégration religieuse et de conservatisme politique mis en évidence par Guy Michelat et Michel Simon. Les seconds, aussi fortement intégrés au catholicisme, ont des attitudes politiques plus progressistes. Ils sont donc proches du modèle minoritaire de Pierre Bréchon et Bernard Denni. Toutefois, les relations sont moins évidentes que prévu. Le MCC apparaît moins conservateur et La Vie nouvelle, moins ancrée à gauche. Des raisons historiques peuvent expliquer ce phénomène surprenant. L'Union sociale des ingénieurs catholiques (USIC), l'ancêtre du MCC, est, à sa création, une institution progressiste. Elle préconise un engagement dans le monde, a des revendications salariales et est parfois virulente envers le patronat, même s'il est catholique. Il semble que le MCC, malgré un changement d'objectif, soit marqué par cette optique «chrétienne-sociale ${ }^{38} »$. Pour sa part, La Vie nouvelle a, lors de sa création, des positions politiques très ancrées à gauche. Mais elle doit, à présent, les relativiser, car elle est insérée à la fois dans le domaine politique et dans le domaine religieux. Or donner trop d'importance à la sphère du politique entraînerait une perte des objectifs religieux et, du coup, elle perdrait sa spécificité. Dans les deux mouvements, on assiste à un indispensable rééquilibrage entre les priorités religieuses et politiques. La dimension religieuse apparaît donc ici comme un facteur qui vient relativiser la dimension politique des mouvements.

Dans un autre ordre d'idée, il pouvait sembler audacieux de postuler l'existence, à l'intérieur d'un groupe religieux bien identifié et intégraliste, d'une diversité des comportements politiques. C'est pourtant l'hypothèse de base de l'article de Christine Pina sur le Renouveau charismatique ${ }^{39}$. À partir d'entretiens dans différentes communautés charismatiques, elle montre la diversité des univers religieux et politiques des membres. Prenant les membres les plus intégrés au Renouveau charismatique - les engagés -, l'auteure retrace leurs itinéraires individuels. Pour deux groupes différents - le Chemin Neuf et les Béatitudes -, elle suit la même démarche: l'exploration des itinéraires de vie. Au Chemin Neuf, la rencontre avec Dieu s'inscrit dans une logique historique et les enquêtés n'évoquent pas de rupture.

${ }^{37}$ A. Grelon et F. Subileau, «Le Mouvement des Cadres Chrétiens et La Vie Nouvelle: des cadres catholiques militants», Revue française de science politique, juin 1989, p. 314-340.

${ }^{38}$ Il s'agit aujourd'hui essentiellement d'un mouvement qui réfléchit sur l'insertion dans le travail. L'orientation syndicale a été abandonnée.

${ }^{39}$ C. Pina, «Religion et politique dans le «Renouveau charismatique». La cas de deux communautés françaises», Religiologiques, no 16, automne 1997, p. 113-133. 
À l'opposé, les engagés des Béatitudes privilégient une vision bipolaire du monde où la rencontre avec Dieu devient le point de départ entre un avant et un après. L'attitude par rapport à la politique est alors tout autre. Au Chemin Neuf, la communauté peut devenir le lieu privilégié pour une expérimentation politique: «Le Chemin Neuf dit qu'un autre chemin d'ordre socio-politique peut être préféré, que le partage économique peut être un choix de société, loin des constructions communistes ou capitalistes.» Aux Béatitudes, le discours sur la politique est très critique. Pour ses membres, la société est malade et le système politique est devenu fou. Dès lors, le rapport à la politique est un tant soit peu délicat. Au sein d'un groupe de catholiques fortement intégralistes, la diversité des conceptions de la société et de la politique peut donc s'observer. Certes, Jean-Marie Donegani l'avait déjà montré, mais ce qui est novateur ici, c'est la spécificité du groupe. Alors que Donegani le montre pour des citoyens français, l'apport de Christine Pina valide ces formes de pluralisme au sein d'un même mouvement, le Renouveau charismatique. Même dans des groupes où le religieux - la dimension émotionnelle, le charisme et l'intuition du groupe, la vision globalisante de la vie - est fortement structurant, des visions différentes du et de la politique se font jour. Si de telles formes de pluralisme existent, on peut alors penser que le pluralisme politique touche tous les groupes, y compris les «virtuoses du religieux».

\section{Conclusion}

$\mathrm{Au}$ terme de cet article, force est de constater que le couple religion et politique est malmené. La sécularisation de la société et la sécularisation interne du christianisme ont eu raison d'une relation monolithique.

Un nouveau paradigme montrant le pluralisme politique des chrétiens est en place. Deux remarques me semblent nécessaires. D'une part, le paradigme du pluralisme n'invalide pas le paradigme précédent, il se substitue à lui en le complétant et en le faisant évoluer. D'autre part, il reste toutefois beaucoup à faire pour valider ce nouveau paradigme. Des études de terrain quantitatives - au moyen de nouveaux indicateurs religieux -- en vue de mesurer ce pluralisme sont nécessaires. C'est la condition de base pour pouvoir notamment mesurer la force sociale des différents modèles établis par Jean-Marie Donegani et Guy Lescanne. Il serait aussi nécessaire de valider ces recherches au moyen d'études comparatives.

Il n'appartient pas au sociologue de prédire la manière dont évoluera le couple religion-politique durant le troisième millénaire. On peut toutefois souligner que la multiplication des groupes religieux - 
charismatique, intégriste, action catholique, etc. - , que l'Église peut difficilement réguler, amène à envisager une multiplicité d'options politiques. De même, la venue de l'islam dans l'espace européen risque d'engendrer de nouvelles configurations du couple religion-politique. Dans une conjoncture où l'islam s'implante durablement et où les fondamentalismes religieux contestent l'autonomie du politique, on peut penser que le couple religion-politique est encore voué à de nombreuses transformations. Le travail du sociologue est donc loin d'être achevé...

Kristoff TALIN

Chargé de recherche au CNRS

CIDSP-IEP Grenoble

\section{Résumé}

Les liens entre religion et politique sont anciens et les études les concernant, relativement nombreuses. En France, le modèle dominant des années soixante - montrant l'intensité de la relation entre vote à droite et intégration au catholicisme - est aujourd'hui remis en question. Les modèles concurrents insistent sur le pluralisme du catholicisme et sur les conséquences politiques plurielles. L'article se propose, d'une part, d'examiner la pertinence de deux modèles, et, d'autre part, de les tester en les appliquant à divers pays d'Europe. Les résultats montrent que la relation entre religion et politique demeure intense et relativement stable, quel que soit le pays étudié ou la religion prise en considération. Ainsi, même si des aménagements au premier paradigme sont nécessaires, il n'en reste pas moins que celui-ci demeure valide pour analyser le couple religion et politique.

Mots-clés: religion, politique, catholicisme, France, Europe, pratique religieuse, vote, droite religieuse.

\section{Summary}

The links between religion and politics have a long history, and studies of them are relatively numerous. In France, the dominant model during the 1960s-which revealed the intensity of the relationship between right-wing voting and integration to Catholicism-is now being questioned. Concurrent models insist on the pluralism of Catholicism and the consequences of plural politics. This article examines the relevance of two models on the one hand, and tests them by applying them to various European countries on the other. It demonstrates that the relationship between religion and politics remains intense and 
relatively stable no matter which country is studied or which religion is considered. As such, despite the need for some adjustments to the first paradigm, it is no less true that this it is still valid for analyzing the religion-politics couplet.

Key-words: religion, politics, Catholicism, France, Europe, religious practice, voting, religious right.

\section{Resumen}

Los lazos entre religión y política son antiguos y los estudios que los conciernen, relativamente numerosos. En Francia, el modelo dominante de los años sesenta - mostrando la intensidad de la relación entre el voto de derecha y la integración al catolicismo - es hoy puesto en duda. Los modelos concurrentes insisten en la pluralidad del catolicismo y en las consecuencias políticas diversas. El artículo se propone, por un lado, examinar la pertinencia de dos modelos, y por el otro someterlos a un test, aplicándolos a distintos países de Europa. Se muestra que la relación entre religión y política se mantiene intensa y relativamente estable, cualquiera sea el país estudiado o la religión puesta en consideración. Así, aún cuando ciertos ajustes se hacen necesarios en el primer modelo, sigue siendo cierto que dicho paradigma es válido aún para analizar el duo religión-política.

Palabras claves: religión, política, catolicismo, Francia, Europa, práctica religiosa, votación, derecha religiosa. 
Tableau 1

Positionnement à gauche sur l'échelle gauche-droite selon la pratique religieuse

\begin{tabular}{|c|c|c|c|c|c|c|c|c|c|c|c|}
\hline . & 1973 & 1976 & 1977 & 1978 & 1980 & 1981 & 1988 & 1989 & 1990 & 1991 & 1992 \\
\hline Moyenne & 33 & 31 & 31 & 32 & 31 & 31 & 35 & 35 & 34 & 33 & 33 \\
\hline $\begin{array}{l}\text { Catholiques } \\
\text { Pratiquants } \\
\text { Non pratiquants }\end{array}$ & $\begin{array}{l}31 \\
52\end{array}$ & $\begin{array}{l}26 \\
53\end{array}$ & $\begin{array}{l}27 \\
49\end{array}$ & $\begin{array}{l}27 \\
51\end{array}$ & $\begin{array}{l}27 \\
45\end{array}$ & $\begin{array}{l}26 \\
47\end{array}$ & $\begin{array}{l}30 \\
45\end{array}$ & $\begin{array}{l}31 \\
50\end{array}$ & $\begin{array}{l}29 \\
49\end{array}$ & $\begin{array}{l}30 \\
47\end{array}$ & $\begin{array}{l}30 \\
41\end{array}$ \\
\hline $\begin{array}{l}\text { Protestants } \\
\text { Pratiquants } \\
\text { Non pratiquants }\end{array}$ & $\begin{array}{l}26 \\
40\end{array}$ & $\begin{array}{l}17 \\
29\end{array}$ & $\begin{array}{l}16 \\
26\end{array}$ & $\begin{array}{l}17 \\
28\end{array}$ & $\begin{array}{l}20 \\
26\end{array}$ & $\begin{array}{l}18 \\
28\end{array}$ & $\begin{array}{l}27 \\
25\end{array}$ & $\begin{array}{l}27 \\
33\end{array}$ & $\begin{array}{l}25 \\
33\end{array}$ & $\begin{array}{l}22 \\
29\end{array}$ & $\begin{array}{l}23 \\
28\end{array}$ \\
\hline $\begin{array}{l}\text { Orthodoxes } \\
\text { Pratiquants } \\
\text { Non pratiquants }\end{array}$ & & & & & & $\begin{array}{l}31 \\
50\end{array}$ & $\begin{array}{l}26 \\
38\end{array}$ & $\begin{array}{l}22 \\
44\end{array}$ & $\begin{array}{l}24 \\
50\end{array}$ & $\begin{array}{l}23 \\
53\end{array}$ & $\begin{array}{l}21 \\
34\end{array}$ \\
\hline Sans-religion & & 49 & 51 & 53 & 48 & 48 & 54 & 52 & 47 & 46 & 47 \\
\hline
\end{tabular}

Les résultats se lisent de la façon suivante: en 1992, $33 \%$ des Européens se positionnent à gauche, mais c'est le cas de $30 \%$ des catholiques pratiquants et de $41 \%$ des non-pratiquants. 
Tableau 2

Odds-ratios droite-gauche entre catholiques pratiquants (CP), protestants pratiquants (PP) et orthodoxes pratiquants (OP) par rapport aux sans-religion (SR)

\begin{tabular}{|l|l|l|l|}
\hline Année & CP/SR & PP/SR & OP/SR \\
\hline $\mathbf{1 9 7 6}$ & 3,9756 & 4,0732 & \\
\hline $\mathbf{1 9 7 7}$ & 4,3684 & 3,5263 & \\
\hline $\mathbf{1 9 7 8}$ & 4,3824 & 3,6765 & \\
\hline $\mathbf{1 9 8 0}$ & 4,7273 & 3,2424 & \\
\hline $\mathbf{1 9 8 1}$ & 5,4615 & 3,3462 & \\
\hline $\mathbf{1 9 8 8}$ & 2,6000 & 2,9250 & 2,8000 \\
\hline $\mathbf{1 9 8 9}$ & 2,5556 & 2,5111 & 2,6222 \\
\hline $\mathbf{1 9 9 0}$ & 3,3429 & 2,4286 & 3,4286 \\
\hline $\mathbf{1 9 9 1}$ & 2,4731 & 2,6237 & 2,3871 \\
\hline $\mathbf{1 9 9 2}$ & 2,1296 & 2,2963 & 1,8889 \\
\hline
\end{tabular}

Ce tableau présente des rapports de pourcentage. Autrement dit, la première colonne présente les résultats des divisions suivantes: [(Pourcentage de catholiques pratiquants votant à droite divisé par le pourcentage de catholiques pratiquants votant à gauche) divisé par le (pourcentage de sans-religion votant à droite divisé par le pourcentage de sansreligion votant à gauche)]. Les colonnes deux et trois appliquent la même formule pour les protestants et pour les orthodoxes. 


\section{Tableau 3}

Positionnement à gauche sur l'échelle gauche-droite selon la pratique religieuse et le pays. Moyenne de la période 1975-1992

\begin{tabular}{|c|c|c|c|c|c|c|c|c|c|c|c|}
\hline & Fr. & Belg. & Pays-B. & All. & Ital. & Dk & Irl. & G.-B. & Grèce & Esp. & Port. \\
\hline Moyenne & 39 & 25 & 34 & 26 & 44 & 26 & 19 & 24 & 28 & 52 & 26 \\
\hline \multicolumn{12}{|l|}{ Catholiques } \\
\hline 1. Pratiquants & 27 & 15 & 20 & 18 & 36 & & 18 & 28 & & 42 & 22 \\
\hline 2. Non pratiquants & 44 & 30 & 33 & 37 & 66 & & 37 & 30 & & 63 & 31 \\
\hline Différence (2-1) & 17 & 15 & 13 & 19 & 30 & & 19 & 12 & & 21 & 9 \\
\hline \multicolumn{12}{|l|}{ Protestants } \\
\hline 1. Pratiquants & 29 & & 19 & 25 & & 19 & & 18 & & & \\
\hline 2. Non pratiquants & 46 & & 39 & 34 & & 30 & & 26 & & & \\
\hline Différence (2-1) & 17 & & 20 & 9 & & 11 & & 8 & & & \\
\hline \multicolumn{12}{|l|}{ Orthodoxes } \\
\hline 1. Pratiquants & & & & & & & & & 25 & & \\
\hline 2. Non pratiquants & & & & & & & & & 52 & & \\
\hline Différence $(2-1)$ & & & & & & & & & 27 & & \\
\hline Sans-religion & 61 & 43 & 49 & 46 & 82 & 42 & 45 & 29 & 86 & 78 & 50 \\
\hline
\end{tabular}

Les résultats se lisent de la façon suivante: en France, durant la période 1975-1992, $39 \%$ des enquêtés se positionnent à gauche, alors que c'est le cas de $27 \%$ des pratiquants et de $44 \%$ des non-pratiquants, soit une différence de $17 \%$, alors que les sans-religion se positionnent à gauche dans une proportion de $61 \%$. 
Tableau 4

Position à gauche en fonction de la pratique religieuse en 1998

\begin{tabular}{|l|c|}
\hline & $\mathbf{1 9 9 8}$ \\
\cline { 2 - 2 } Moyenne européenne & 36 \\
\hline Catholiques & 31 \\
$\quad$ Pratiquants & 44 \\
$\quad$ Non pratiquants & 29 \\
\hline Protestants & 37 \\
$\quad$ Pratiquants & 22 \\
$\quad$ Non pratiquants & 37 \\
\hline Orthodoxes & 45 \\
$\quad$ Pratiquants & \\
\hline Non pratiquants & \\
\hline Sans-religion & \\
\hline
\end{tabular}

Le tableau se lit de la façon suivante: $36 \%$ des Européens en 1998 se positionnent à gauche, alors que c'est le cas de $31 \%$ des catholiques pratiquants et de $44 \%$ des catholiques non pratiquants. 
Tableau 5

Positionnement à gauche sur l'échelle gauche-droite selon la pratique en fonction des pays en 1998

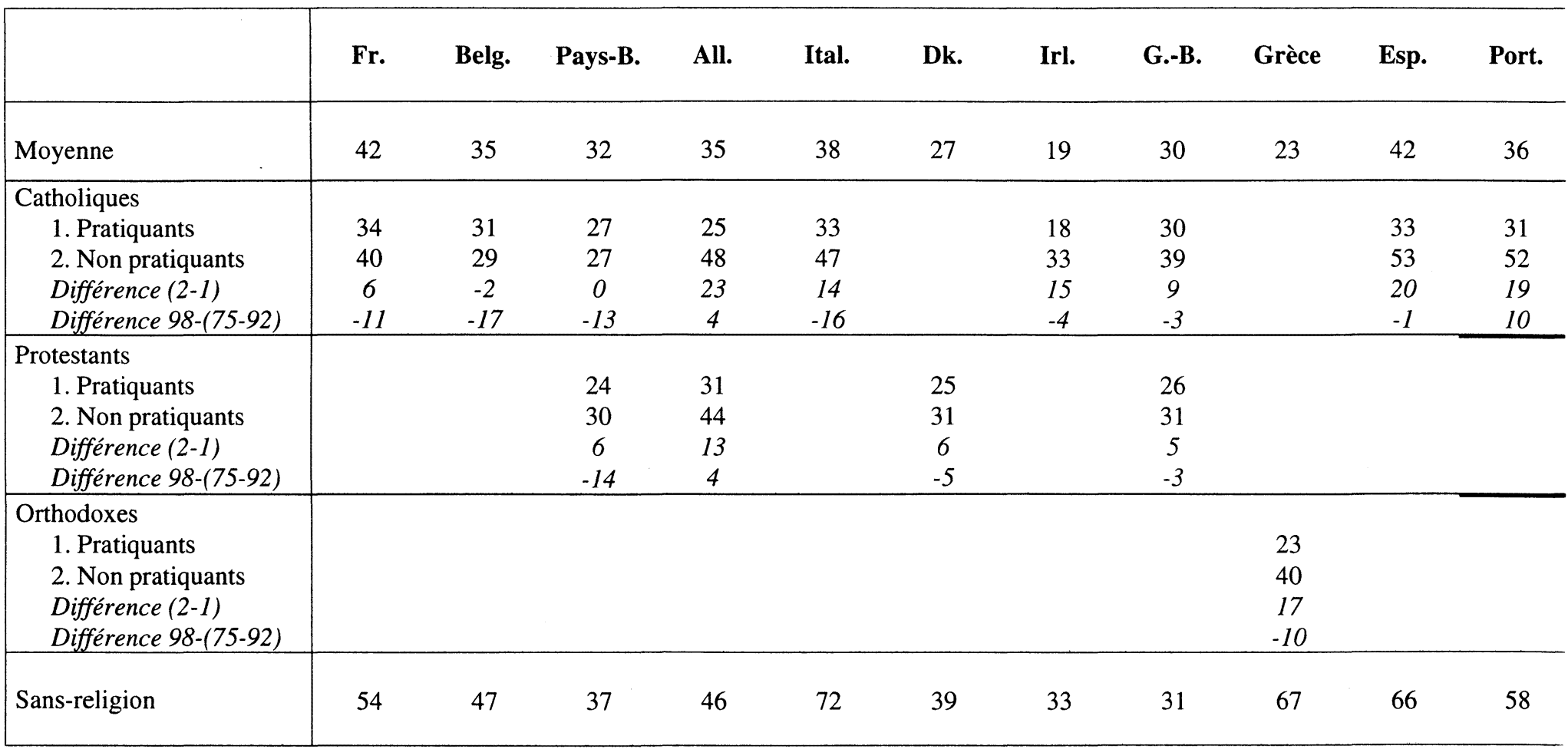

Les résultats se lisent de la façon suivante: en France, en 1998, $42 \%$ des enquêtés se positionnent à gauche, alors que c'est le cas de $34 \%$ des pratiquants et de $40 \%$ des non-pratiquants, soit une différence de $6 \%$. La différence entre 1998 et la moyenne de la période $1975-1992$ est de $-11 \%$, ce qui signifie que l'écart entre pratiquants et non-pratiquants tend à diminuer dans le temps. 